\title{
CATACLYSMIC VARIABLES FROM SLOAN DIGITAL SKY SURVEY. IV. THE FOURTH YEAR (2003) ${ }^{1}$
}

\author{
Paula Szkody, ${ }^{2}$ Arne Henden, ${ }^{3,4}$ Oliver J. Fraser, ${ }^{2}$ Nicole M. Silvestri, ${ }^{2}$ Gary D. Schmidt, ${ }^{5}$ John J. Bochanski, $^{2}$ \\ Michael A. Wolfe, ${ }^{2}$ Marcel Agüeros, ${ }^{2}$ Scott F. Anderson, ${ }^{2}$ Lee Mannikko, ${ }^{2}$ \\ Ronald A. Downes, ${ }^{6}$ Donald P. Schneider, ${ }^{7}$ and J. Brinkmann ${ }^{8}$ \\ Received 2005 January 3; accepted 2005 February 10
}

\begin{abstract}
This paper continues the series presenting cataclysmic variables identified during the fourth year (2003) of the Sloan Digital Sky Survey. We describe 44 close binary systems, of which 12 are the previously known systems EN Cet, EG Cnc, V844 Her, GG Leo, Leo 7, CY UMa, DV UMa, EK UMa, EV UMa, UMa 8, EUVE J0854+390, and RX J0859.1+0537 and 32 are new cataclysmic variables. As in the previous papers, the positions, colors, and spectra of all 44 systems are shown, along with follow-up spectroscopic, photometric, and/or polarimetric observations of 15 of the new systems. The new objects include one eclipsing system, eight with prominent He II emission (of which two are confirmed new polars), and 12 systems showing features of the underlying stars. Our spectropolarimetry also confirms EUVE J0854+390 as a polar.
\end{abstract}

Key words: binaries: eclipsing — binaries: spectroscopic — dwarf novae — novae, cataclysmic variables

\section{INTRODUCTION}

The Sloan Digital Sky Survey (SDSS) data releases have now included the Early Data Release (EDR; Stoughton et al. 2002) and Data Releases 1, 2, and 3 (DR1, DR2, DR3; Abazajian et al. 2003, 2004, 2005 ${ }^{9}$ ). In parallel with these releases, we are publishing the cataclysmic variables (CVs) for which SDSS spectra have been obtained. (A comprehensive review of CVs and all their different subtypes can be found in Warner 1995.) Our presentations are by yearly increments, so the results cover slightly more plates than the releases. Our previous three papers have identified 19 new CVs from spectra through 2000 December 31 (Szkody et al. 2002, hereafter Paper I), 35 in 2001 (Szkody et al. 2003b, hereafter Paper II), and 34 in 2002 (Szkody et al. 2004, hereafter Paper III). This paper continues the series with 27 new CVs found in spectra obtained in 2003, as well as five systems that were observed in previous years but missed in past manual searches. In addition, $12 \mathrm{CVs}$ had spectra taken in 2003 that were previously known.

In order to determine the nature of the CVs that are being found in the SDSS, which samples to fainter magnitudes and/or larger areas than previous sky surveys, follow-up photometry and spectroscopy are needed to determine the fundamental parameters of orbital periods, inclination, and magnetic fields. We have begun this work on 15 systems and present the results so that more detailed studies can be performed on these and the rest of the sample. Once enough information is available, com-

\footnotetext{
${ }^{1}$ Based on observations obtained with the Sloan Digital Sky Survey and with the Apache Point Observatory (APO) $3.5 \mathrm{~m}$ telescope, which are owned and operated by the Astrophysical Research Consortium (ARC).

2 Department of Astronomy, University of Washington, Box 351580, Seattle, WA 98195.

${ }^{3}$ US Naval Observatory, Flagstaff Station, P.O. Box 1149, Flagstaff, AZ 86002-1149.

${ }^{4}$ Universities Space Research Association.

5 University of Arizona, Steward Observatory, Tucson, AZ 85721.

6 Space Telescope Science Institute, 3700 San Martin Drive, Baltimore, MD 21218.

7 Department of Astronomy and Astrophysics, 525 Davey Laboratory, Pennsylvania State University, University Park, PA 16802.

8 Apache Point Observatory, P.O. Box 59, Sunspot, NM 88349.

${ }^{9}$ See http://www.sdss.org.
}

parisons can be made with population models (e.g., Howell et al. 2001) to determine correct evolutionary scenarios for close binaries. Gänsicke (2005) provides a preliminary comparison of SDSS statistics with other surveys. Our follow-up is primarily designed to aid in identifying the type of $\mathrm{CV}$ and to determine if the period is likely to be long or short, i.e., below or above the $2-3 \mathrm{hr}$ period gap. The intent is to provide a starting point for more detailed follow-up studies of individual systems that may be of interest to the community.

\section{OBSERVATIONS AND REDUCTIONS}

The details of the SDSS imaging and spectroscopic instrumentation and reductions can be found in Paper I and in the papers by Fukugita et al. (1996), Gunn et al. (1998), Lupton et al. (1999, 2001), York et al. (2000), Hogg et al. (2001), Smith et al. (2002), Pier et al. (2003), and Ivezic et al. (2004). As a brief summary, SDSS photometry in five filters $(u, g, r, i$, and $z)$ is used by a pipeline that selects objects by color for later spectroscopy in the range of 3900-6200 $\AA$ (blue beam) and 5800$9200 \AA$ (red beam) at a resolving power of $\sim 1800$. All spectra are calibrated for wavelength and flux and then classified as stars, galaxies, and quasars. CVs obtain spectra through several color selection algorithms, since their colors overlap with those of hot stars, quasars, white dwarfs, and M stars, depending on how much the accretion disk or accretion column contributes to the optical light over that of the underlying white dwarf and latetype secondary star (see Papers I and II for further explanation). Since Paper II, we have used a computer program that selects objects with Balmer and helium emission/absorption to be hand identified as white dwarfs or CVs. During the past year, we have used this program to examine all the past plates and were able to recover five new CVs that were missed in the past manual searches because of either missed plates or missed identifications. We include these here along with the 39 objects identified from 2003 plates.

The 32 new CVs and the 12 previously known objects are listed in Table 1 with the plate, fiber, and modified Julian date (MJD) of each spectrum. The five objects recovered from previous years have MJD $<52,600$. The magnitudes and colors are from the point-spread function photometry with no correction for interstellar reddening. For simplicity, we refer to 
TABLE 1

Cataclysmic Variables with SDSS Spectra

\begin{tabular}{|c|c|c|c|c|c|c|c|c|}
\hline SDSS $J^{\mathrm{a}}$ & MJD-P-F ${ }^{b}$ & $g$ & $u-g$ & $g-r$ & $r-i$ & $i-z$ & $\begin{array}{c}P \\
(\mathrm{hr})\end{array}$ & Comments $^{\mathrm{c}}$ \\
\hline $001856.93+345444.3 \ldots \ldots \ldots$. & $52999-1468-163$ & 17.82 & 0.37 & 0.52 & 0.18 & 0.15 & $\ldots$ & He II \\
\hline 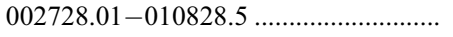 & $52933-1493-204$ & 20.72 & -0.04 & 0.04 & 0.09 & 0.29 & $\ldots$ & EN Cet \\
\hline 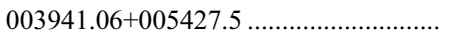 & $52261-0690-568$ & 20.57 & 0.00 & -0.15 & -0.41 & 0.24 & $\cdots$ & $\cdots$ \\
\hline $005050.88+000912.7 \ldots \ldots \ldots \ldots \ldots \ldots \ldots \ldots \ldots$ & $52883-1496-493$ & 20.38 & -0.05 & 0.02 & -0.13 & -0.34 & $\ldots$ & $\ldots$ \\
\hline 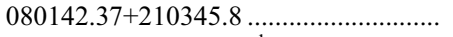 & $52941-1583-595$ & 18.84 & -0.43 & 0.18 & 0.16 & 0.11 & $\ldots$ & $\ldots$ \\
\hline $080303.90+251627.0^{* \mathrm{~d}}$. & $52670-1205-188$ & 19.59 & -0.43 & 0.24 & 0.29 & 0.30 & 1.7 & $\ldots$ \\
\hline $081352.02+281317.3^{*} \ldots \ldots$ & $52670-1206-380$ & 17.16 & -0.08 & -0.05 & 0.02 & -0.07 & 2.7 & He II \\
\hline $083751.00+383012.5^{*} \ldots \ldots \ldots \ldots \ldots \ldots \ldots \ldots \ldots \ldots \ldots \ldots \ldots \ldots \ldots$ & $52317-0828-049$ & 19.13 & 0.03 & 0.02 & 0.52 & 0.68 & $\cdots$ & Polar \\
\hline $083931.35+282824.0 \ldots \ldots \ldots$ & $52965-1588-294$ & 20.24 & -0.20 & 0.06 & 0.11 & 0.55 & $\ldots$ & $\ldots$ \\
\hline 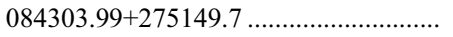 & $52965-1588-278$ & 18.87 & 0.16 & -0.02 & -0.19 & -0.04 & 1.41 & $\mathrm{EG} \mathrm{Cnc}$ \\
\hline $085414.02+390537.3^{*} \ldots \ldots \ldots \ldots \ldots \ldots \ldots \ldots \ldots \ldots \ldots \ldots \ldots \ldots$ & $52703-1199-286$ & 19.17 & 0.54 & -0.05 & 0.00 & 0.15 & $\ldots$ & EUVE J0854+390 polar \\
\hline 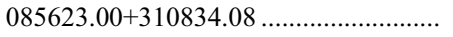 & $52974-1271-088$ & 19.99 & 0.20 & 0.01 & -0.25 & -0.06 & $\ldots$ & $\ldots$ \\
\hline $085909.18+053654.5^{*} \ldots \ldots \ldots$ & $52649-1192-343$ & 18.59 & 0.17 & 0.10 & 0.22 & 0.34 & 1.1 & RX J0859 polar \\
\hline 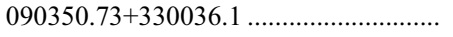 & $52989-1272-188$ & 18.84 & 0.05 & 0.05 & -0.08 & 0.02 & 1.3 & $\ldots$ \\
\hline 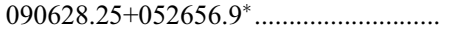 & 52674-1191-007 & 18.76 & 0.06 & 0.30 & 0.37 & 0.28 & $\ldots$ & $\mathrm{DN}$ \\
\hline 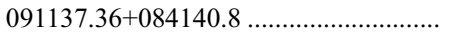 & $52976-1301-278$ & 19.73 & -0.11 & 0.57 & 0.50 & 0.36 & $\cdots$ & $\cdots$ \\
\hline $091935.66+502825.2^{*} \ldots \ldots$. & $52247-0766-517$ & 19.88 & -0.08 & 0.68 & 0.77 & 0.26 & $\ldots$ & $\ldots$ \\
\hline 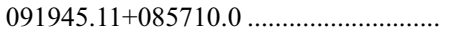 & $52976-1301-624$ & 18.20 & 0.16 & -0.04 & -0.19 & -0.11 & 1.4 & $\ldots$ \\
\hline 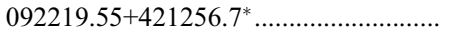 & $52674-1201-075$ & 19.86 & -0.01 & -0.25 & 0.00 & 0.23 & $\ldots$ & $\ldots$ \\
\hline $092229.26+330743.6 \ldots \ldots \ldots \ldots \ldots \ldots \ldots \ldots \ldots \ldots$ & $52990-1592-593$ & 18.44 & -0.30 & -0.03 & 0.03 & 0.24 & $\ldots$ & $\ldots$ \\
\hline 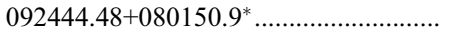 & $52724-1195-403$ & 19.26 & 0.24 & -0.01 & 0.57 & 0.81 & 2 & He II \\
\hline 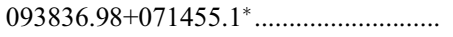 & $52733-1196-026$ & 18.32 & -0.04 & 0.54 & 0.72 & 0.60 & 4.48 & Leo 7 (PG 0935+075) \\
\hline $094636.60+444644.7^{*} \ldots \ldots$. & $52672-1202-030$ & 19.38 & -0.02 & 0.23 & 0.35 & 0.58 & 2.06 & DV UMa \\
\hline $101534.67+090442.1^{*} \ldots \ldots \ldots \ldots \ldots \ldots \ldots \ldots \ldots$ & $52762-1237-510$ & 17.22 & -0.15 & 0.34 & 0.00 & -0.07 & 1.33 & GG Leo polar \\
\hline 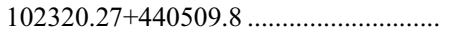 & $52990-1429-359$ & 18.83 & -0.31 & 0.14 & 0.11 & 0.06 & $\ldots$ & UMa 8 (NSV 4838) \\
\hline 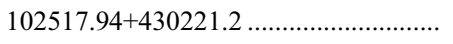 & $52994-1358-077$ & 19.94 & 0.08 & 0.06 & -0.46 & 0.12 & $\cdots$ & $\cdots$ \\
\hline $103147.99+085224.3^{*} \ldots \ldots$ & $52760-1239-621$ & 18.79 & 0.00 & 0.05 & 0.19 & 0.28 & $\ldots$ & $\ldots$ \\
\hline 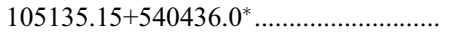 & $52652-1011-345$ & 18.39 & 0.03 & -0.49 & -0.06 & -0.12 & 1.91 & EK UMa polar \\
\hline 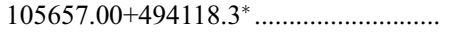 & $52669-0876-103$ & 17.78 & -0.27 & 0.25 & 0.18 & 0.16 & 1.63 & CY UMa \\
\hline 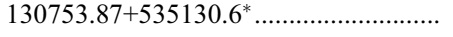 & $52707-1039-069$ & 16.53 & 0.34 & -0.33 & -0.06 & -0.08 & 1.33 & EV UMa polar \\
\hline $133941.12+484727.5^{*} \ldots \ldots \ldots \ldots \ldots \ldots \ldots \ldots$ & $52762-1283-591$ & 17.64 & 0.19 & -0.10 & -0.13 & -0.14 & $\ldots$ & $\ldots$ \\
\hline $142955.86+414516.8^{*} \ldots \ldots \ldots \ldots \ldots \ldots \ldots \ldots \ldots \ldots \ldots \ldots \ldots \ldots \ldots$ & $52825-1395-045$ & 17.74 & -0.38 & -0.12 & 0.07 & 0.04 & 1.4 & He II \\
\hline $145758.21+514807.9^{*} \ldots \ldots \ldots \ldots \ldots \ldots \ldots \ldots$ & $52786-1328-618$ & 19.54 & -0.10 & 0.04 & -0.13 & 0.08 & $\ldots$ & $\ldots$ \\
\hline $150722.33+523039.8^{*} \ldots \ldots \ldots \ldots \ldots \ldots \ldots \ldots \ldots \ldots \ldots \ldots \ldots \ldots$ & $52703-1165-372$ & 18.36 & 0.12 & -0.10 & -0.17 & 0.17 & 1.12 & $\mathrm{Ec}$ \\
\hline 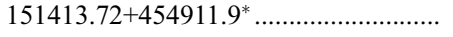 & $52721-1050-171$ & 19.68 & 0.34 & 0.00 & -0.16 & -0.14 & $\ldots$ & $\ldots$ \\
\hline $153634.42+332851.9^{*} \ldots \ldots \ldots \ldots \ldots \ldots \ldots \ldots \ldots \ldots \ldots \ldots \ldots \ldots \ldots$ & $52823-1355-054$ & 19.20 & -0.24 & 0.25 & 0.17 & 0.18 & $\ldots$ & $\ldots$ \\
\hline $154104.67+360252.9 \ldots \ldots \ldots$. & $52872-1402-445$ & 19.70 & 0.22 & -0.13 & 0.52 & 0.04 & 1.4 & Polar H, L \\
\hline 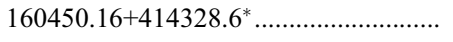 & $52824-1335-531$ & 17.67 & -0.01 & -0.17 & -0.06 & -0.19 & $\cdots$ & He II NP \\
\hline $162501.75+390926.4^{*} \ldots \ldots \ldots \ldots \ldots \ldots \ldots \ldots$ & $52759-1172-212$ & 17.20 & -0.09 & 0.09 & 0.12 & 0.16 & 1.31 & V844 Her \\
\hline 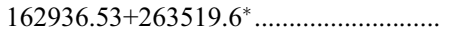 & $52824-1409-339$ & 19.26 & -0.22 & 0.51 & 0.60 & 0.47 & 2.03 & He II \\
\hline 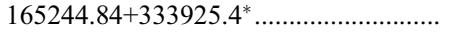 & $52791-1175-617$ & 18.84 & 0.52 & -0.12 & -0.13 & -0.03 & $\ldots$ & $\ldots$ \\
\hline 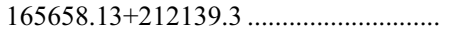 & $52880-1415-095$ & 18.52 & -0.49 & 0.23 & 0.10 & 0.13 & $\ldots$ & $\ldots$ \\
\hline $214354.60+124458.0^{*} \ldots \ldots \ldots \ldots \ldots \ldots \ldots \ldots \ldots \ldots \ldots \ldots \ldots \ldots$ & $52221-0732-589$ & 16.19 & 0.69 & 0.07 & -0.04 & 0.05 & $\ldots$ & $\ldots$ \\
\hline 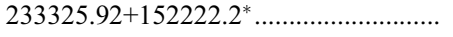 & $52238-0746-614$ & 18.75 & -0.42 & 0.33 & -0.02 & 0.00 & 1.4 & $\ldots$ \\
\hline
\end{tabular}

a Objects marked with asterisks are publicly available in the SDSS DR3 or DR2.

b MJD-Plate-Fiber for spectra.

c DN is a dwarf nova, Ec is eclipsing, NP is not polarized, and $\mathrm{H}$ and $\mathrm{L}$ show high and low states.

d Object is the southwestern star of a close pair.

these objects throughout the rest of this paper as SDSSJ hhmm (where hhmm is the right ascension in hours and minutes) except for the four systems that have the same first four digits of right ascension as others, so we add the first two digits of declination to distinguish them.

Follow-up observations on several systems were performed to refine their characteristics (estimate their orbital period, whether they were at a high enough inclination to be eclipsing, and whether they show polarization). Differential photometry was accomplished with the US Naval Observatory Flagstaff Station (NOFS) $1 \mathrm{~m}$ telescope, using a $1024 \times 1024$ SITe/Tektronix CCD with no filter in order to maximize the signal-to-noise ratio $(\mathrm{S} / \mathrm{N})$. Previous nights of calibrated all-sky photometry with Landolt standards were used to calibrate the comparison stars and place the photometry onto the Johnson $V$-magnitude system.

Time-resolved spectra were obtained at the APO $3.5 \mathrm{~m}$ telescope with the Double Imaging Spectrograph in highresolution mode (resolution about $3 \AA$ ) and a 1 ".5 slit. The reductions were the same as those used in the previous papers, with the line velocities measured with either the "e" (which determines the centroid of the line) or " $k$ " (which fits a Gaussian to the line) in the IRAF ${ }^{10}$ SPLOT package (for simple or

\footnotetext{
${ }^{10}$ IRAF (Image Reduction and Analysis Facility) is distributed by the National Optical Astronomy Observatory, which is operated by the Association of Universities for Research in Astronomy, Inc., under cooperative agreement with the National Science Foundation (NSF).
} 
TABLE 2

Follow-UP Data

\begin{tabular}{|c|c|c|c|c|c|}
\hline SDSS J & UT Date & Site & Time (UT) & $\begin{array}{l}\text { Exposure } \\
\text { (s) }\end{array}$ & Data Obtained \\
\hline $0018 \ldots$ & 2004 Sep 21 & Bok & $8: 13-8: 57$ & $3 \times 800$ & Polarimetry $<0.2 \%$ \\
\hline $0018 \ldots$ & 2004 Oct 10 & $\mathrm{APO}$ & $2: 41-4: 29$ & 600 & 11 spectra \\
\hline 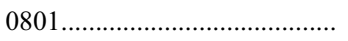 & 2004 Feb 20 & APO & $3: 43-6: 13$ & 1200 & 7 spectra \\
\hline 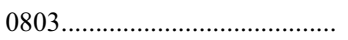 & 2003 Dec 29 & APO & $5: 10-7: 01$ & 600,900 & 7 spectra \\
\hline 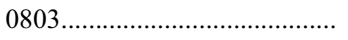 & 2004 Jan 27 & NOFS & $3: 08-12: 08$ & 180 & Phot \\
\hline 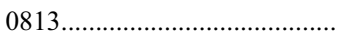 & 2003 Dec 21 & APO & $5: 29-8: 33$ & 600 & 16 spectra \\
\hline 0813 & 2004 Jan 30 & NOFS & $2: 56-8: 59$ & 80 & Phot \\
\hline 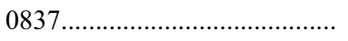 & 2004 Apr 17 & APO & $2: 43-2: 58$ & 900 & 1 spectrum \\
\hline $0854 \ldots \ldots \ldots \ldots \ldots \ldots \ldots \ldots . .$. & 2004 Dec 18 & MMT & $8: 41-9: 08$ & $3 \times 480$ & Polarimetry \\
\hline 0903 & 2003 Dec 30 & APO & $4: 50-7: 15$ & 900,1200 & 6 spectra \\
\hline 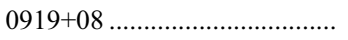 & 2004 Apr 17 & APO & $3: 08-5: 05$ & 600 & 11 spectra \\
\hline 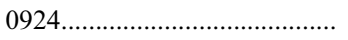 & 2003 Dec 21 & APO & $8: 49-10: 48$ & 600 & 11 spectra \\
\hline $0924 \ldots .$. & 2004 May 4 & NOFS & $3: 15-6: 59$ & 120 & Phot \\
\hline $0924 \ldots$. & 2004 Dec 12 & MMT & $9: 55-10: 34$ & 2160 & Polarimetry $<0.4 \%$ \\
\hline 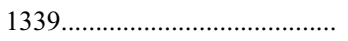 & 2004 Mar 18 & APO & $8: 30-10: 22$ & 900 & 7 spectra \\
\hline 1339 & 2004 Apr 27 & NOFS & $2: 52-11: 26$ & 80 & Phot \\
\hline $1429 \ldots \ldots \ldots \ldots \ldots \ldots \ldots . .$. & 2004 Feb 20 & APO & $9: 32-11: 53$ & 900,1200 & 7 spectra \\
\hline 1429 & 2004 Apr 26 & NOFS & $4: 23-11: 23$ & 200 & Phot \\
\hline $1507 \ldots \ldots \ldots \ldots$ & 2003 Sep 21 & APO & $2: 22-4: 32$ & 1200,1500 & 5 spectra \\
\hline 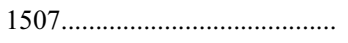 & 2004 May 10 & NOFS & $6: 54-11: 28$ & 140 & Phot \\
\hline $1541 \ldots \ldots \ldots \ldots \ldots \ldots \ldots \ldots \ldots . .$. & 2004 Feb 15 & MMT & 10:07-10:41 & $2 \times 960$ & Polarimetry \\
\hline 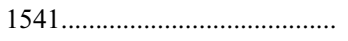 & 2004 May 17 & NOFS & $7: 35-11: 31$ & 200 & Phot \\
\hline 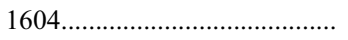 & 2003 Oct 31 & MMT & $2: 07-2: 36$ & $2 \times 800$ & Polarimetry $<0.2 \%$ \\
\hline 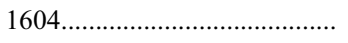 & 2004 Feb 15 & MMT & $12: 26-13: 00$ & $2 \times 960$ & Polarimetry $<0.1 \%$ \\
\hline 1629 & 2003 Sep 27 & APO & $2: 15-4: 01$ & 600,900 & 7 spectra \\
\hline 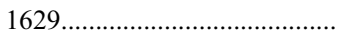 & 2004 May 23 & NOFS & $5: 14-11: 14$ & 260 & Phot \\
\hline 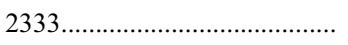 & 2004 Sep 7 & NOFS & $3: 31-12: 17$ & 140 & Phot \\
\hline 2333 & 2004 Sep 13 & APO & $3: 21-6: 52$ & 600 & 19 spectra \\
\hline
\end{tabular}

narrow-line structure) or with a double-Gaussian method (Shafter 1983) for lines with central absorption or narrow emission components within the broad emission. A least-squares procedure was used to fit a sinusoid to the velocities in order to determine $\gamma$ (systemic velocity), $K$ (semiamplitude), $P$ (orbital period), and $\phi_{0}$ (phase of crossing from redshift to blueshift).

Circular polarization was measured for six systems, using the CCD Spectropolarimeter SPOL with a low-resolution grating on the $6.5 \mathrm{~m}$ MMT on Mount Hopkins or the $2.3 \mathrm{~m}$ Bok Telescope on Kitt Peak. The dates and types of follow-up observations are summarized in Table 2.

\section{RESULTS}

The SDSS spectra for all 44 systems are shown in Figure 1, and Table 3 gives the equivalent widths and fluxes of the prominent hydrogen Balmer and helium emission lines. From the spectral appearance and the available follow-up data, we were able to categorize the CVs as described below.

\subsection{Previously Known Systems}

Twelve of the CVs with spectra obtained during 2003 are previously known. These include EN Cet, EG Cnc, V844 Her, GG Leo, Leo 7 (PG 0935+075), CY UMa, DV UMa, EK UMa, EV UMa, UMa 8 (NSV 4838), EUVE J0854+390, and RX J0859.1+ 0537 (see Table 1 for the SDSS designations). Information on these 12 sources is available from the online $\mathrm{CV}$ catalog of Downes et al. (2001). ${ }^{11}$ Four (GG Leo, EK UMa, EV UMa, and RX J0859.1+0537) of the 12 known objects are polars, i.e., systems containing a white dwarf with a magnetic

\footnotetext{
${ }^{11}$ See http://icarus.stsci.edu/ downes/cvcat.
}

field over $10 \mathrm{MG}$ (Wickramasinghe \& Ferrario 2000). All four appear to have been in relatively high states of accretion at the times of the SDSS spectra, as evidenced by the strength of their He II emission.

The SDSS spectrum of EUVE J0854+390 shows some He II, is an X-ray source (see $\S 3.6$ ), and has a spectrum brighter than implied by the photometry (Table 1). Christian et al. (2001) showed three spectra of this source that also reveal large variability. Their spectra all have about a factor of 10 larger flux than the SDSS spectrum and have much stronger He II, implying a higher state of accretion at that time. The velocities in their three spectra also showed large shifts $\left(400 \mathrm{~km} \mathrm{~s}^{-1}\right)$. They suggested that EUVE J0854+390 is probably a polar with an orbital period of $<2 \mathrm{hr}$. Our spectropolarimetry, obtained in an accretion state similar to that of the SDSS, confirms the magnetic nature of this object. Three observations spanning approximately one-quarter of a $\sim 2 \mathrm{hr}$ orbital period display circular polarization reaching $-30 \%$ and reveal cyclotron harmonic features in the total flux. Figure 2 shows the first and third observations from the sequence. The harmonics appear in the third observation, presumably because the accretion funnel has become roughly perpendicular to our view (see Wickramasinghe \& Ferrario 2000 for a discussion of cyclotron beaming). Using the observed peaks to derive the magnetic field strength and temperature yields a corresponding fit for the harmonics shown in the lower right of Figure 2 for $B=44 \mathrm{MG}$ and $T \sin ^{2} \theta=16 \mathrm{keV}$.

The spectra of EG Cnc, V844 Her, Leo 7, CY UMa, and DV UMa are similar to past published spectra of these objects. V844 Her and CY UMa show the strong blue continuum and emission lines characteristic of high accretion rate systems. EG Cnc shows the prominent absorption from the white dwarf 

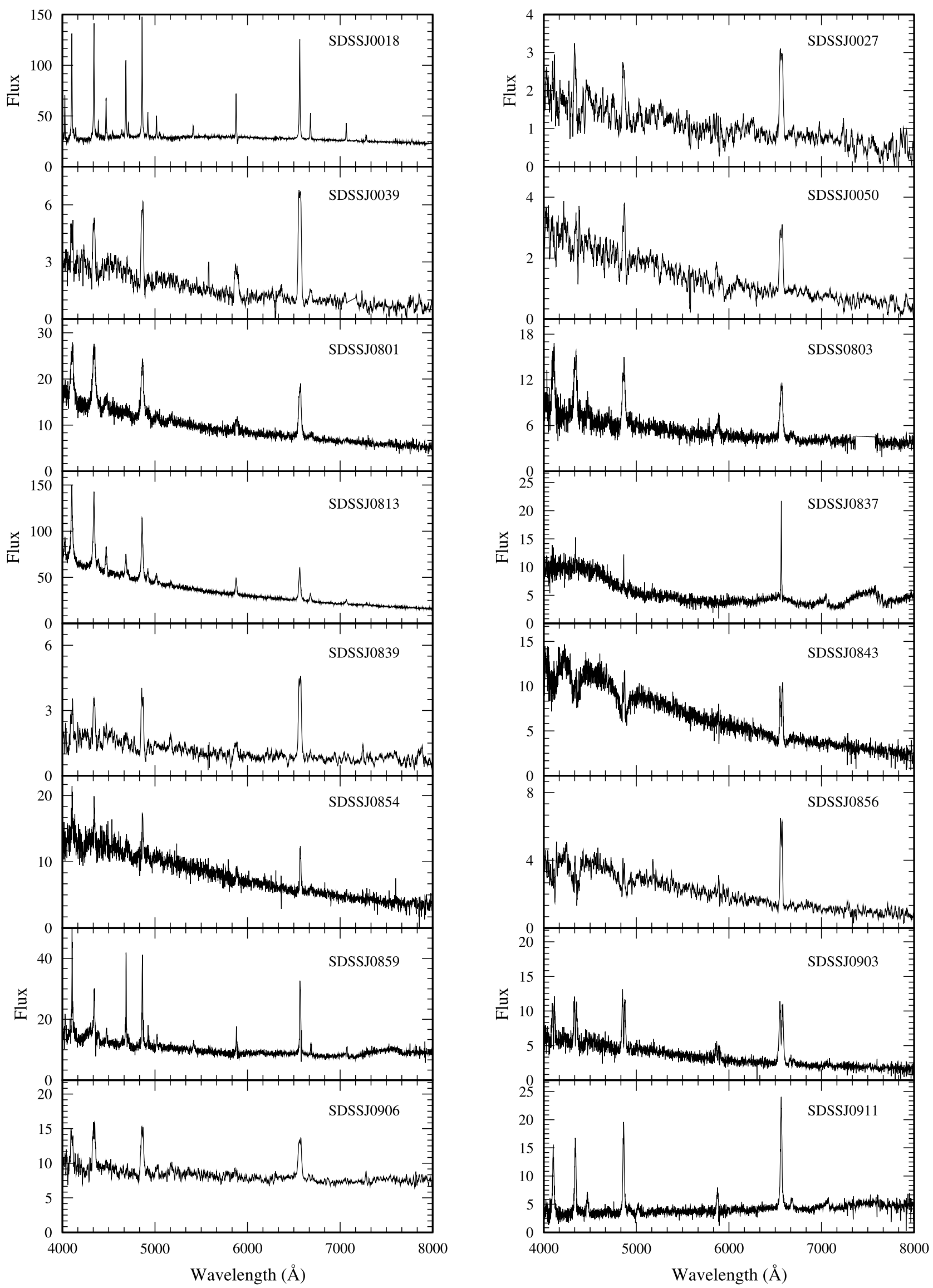

FIG. 1.-SDSS spectra of the 44 CVs. The flux scale is in units of flux density in $10^{-17} \mathrm{ergs} \mathrm{cm}^{-2} \mathrm{~s}^{-1} \AA^{-1}$. The spectral resolution is about $3 \AA$. 

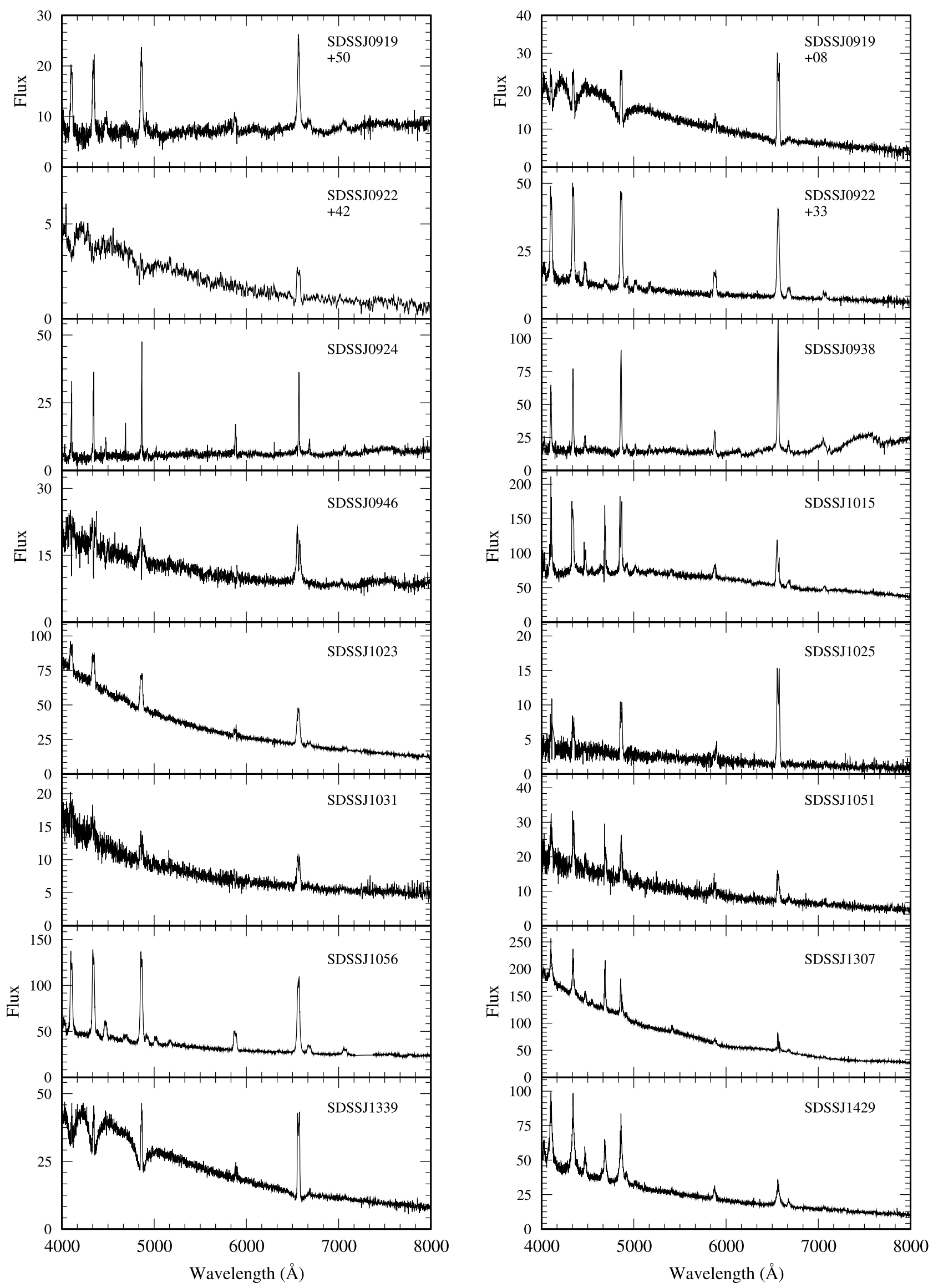

Fig. 1._Continued 

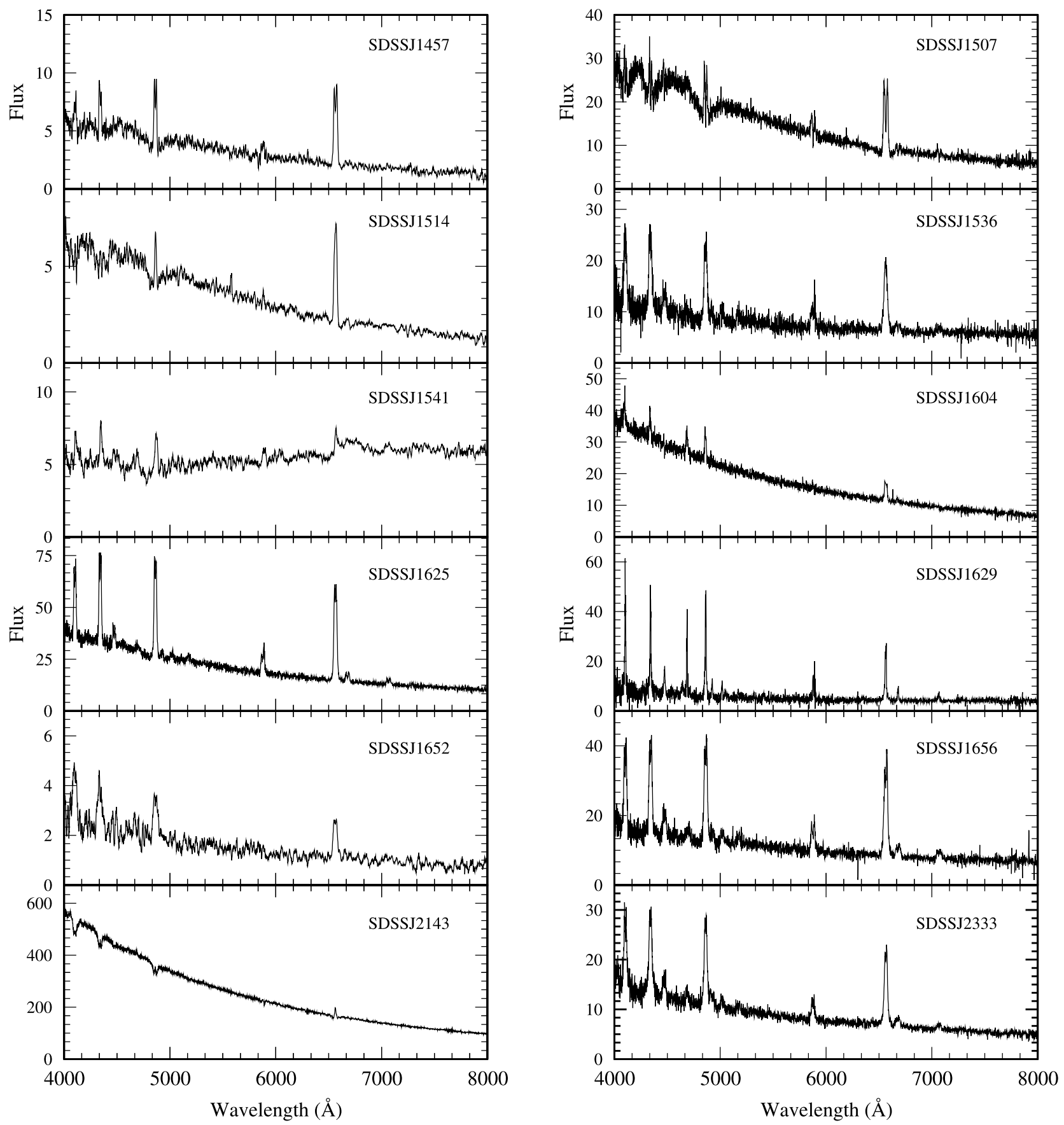

FIg. 1.-Continued 
TABLE 3

SDSS Emission-Line Fluxes and Equivalent Widths ${ }^{\mathrm{a}}$

\begin{tabular}{|c|c|c|c|c|c|c|c|c|}
\hline \multirow[b]{2}{*}{ SDSS $\mathrm{J}$} & \multicolumn{2}{|c|}{$\mathrm{H} \beta$} & \multicolumn{2}{|c|}{$\mathrm{H} \alpha$} & \multicolumn{2}{|c|}{ He 4471} & \multicolumn{2}{|c|}{ He II 4686} \\
\hline & Flux & EW & Flux & EW & Flux & EW & Flux & EW \\
\hline 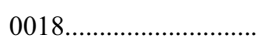 & 11 & 33 & 12 & 42 & 3.4 & 12 & 6.8 & 22 \\
\hline $0027 \ldots$ & 0.5 & 40 & 1.0 & 114 & $\ldots$ & $\ldots$ & $\ldots$ & $\ldots$ \\
\hline 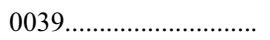 & 1.1 & 58 & 2.2 & 221 & $\ldots$ & $\ldots$ & $\ldots$ & $\ldots$ \\
\hline 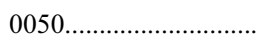 & 0.4 & 20 & 0.9 & 91 & $\ldots$ & $\ldots$ & $\ldots$ & $\ldots$ \\
\hline $0801 \ldots \ldots \ldots$ & 3.5 & 28 & 3.5 & 46 & 0.7 & 5.4 & $\ldots$ & $\ldots$ \\
\hline 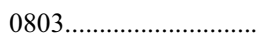 & 2.8 & 46 & 2.4 & 54 & 0.6 & 8.4 & $\ldots$ & $\ldots$ \\
\hline 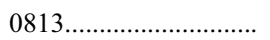 & 13 & 26 & 7.9 & 30 & 4.1 & 7.2 & 4.0 & 7.7 \\
\hline 0837.................................... & 0.3 & 3.9 & 1.2 & 26 & $\ldots$ & $\ldots$ & $\ldots$ & $\ldots$ \\
\hline 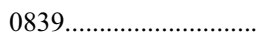 & 0.7 & 57 & 1.4 & 169 & $\ldots$ & $\ldots$ & $\ldots$ & $\ldots$ \\
\hline 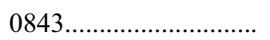 & $\ldots$ & $\ldots$ & 2.0 & 53 & $\ldots$ & $\ldots$ & $\ldots$ & $\ldots$ \\
\hline $0854 \ldots \ldots$ & 1.0 & 9.3 & 1.1 & 20 & $\ldots$ & $\ldots$ & $\ldots$ & $\ldots$ \\
\hline 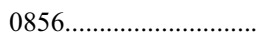 & 0.4 & 18 & 1.4 & 107 & $\ldots$ & $\ldots$ & $\ldots$ & $\ldots$ \\
\hline 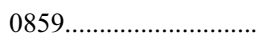 & 3.5 & 30 & 3.7 & 42 & 0.6 & 4.8 & 2.0 & 16 \\
\hline 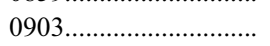 & 2.5 & 56 & 3.8 & 153 & $\ldots$ & $\ldots$ & $\ldots$ & $\ldots$ \\
\hline 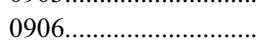 & 2.2 & 25 & 2.2 & 26 & $\cdots$ & $\cdots$ & $\cdots$ & $\cdots$ \\
\hline 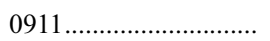 & 3.0 & 76 & 3.8 & 77 & 0.5 & 15 & $\ldots$ & $\ldots$ \\
\hline 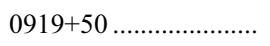 & 4.1 & 57 & 4.9 & 58 & 0.7 & 9.6 & $\ldots$ & $\ldots$ \\
\hline 0919+08 …..................... & $\ldots$ & $\ldots$ & 6.7 & 110 & $\ldots$ & $\ldots$ & $\ldots$ & $\ldots$ \\
\hline $0922+42 \ldots \ldots \ldots \ldots \ldots \ldots$ & $\ldots$ & $\ldots$ & 0.8 & 95 & $\ldots$ & $\ldots$ & $\ldots$ & $\ldots$ \\
\hline $0922+33 \ldots \ldots \ldots \ldots$ & 9.6 & 76 & 9.7 & 116 & 1.9 & 14 & 0.6 & 5.1 \\
\hline 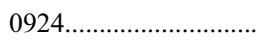 & 2.9 & 49 & 2.9 & 41 & 0.8 & 15 & 0.9 & 17 \\
\hline 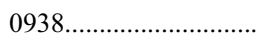 & 12 & 82 & 18 & 111 & 1.9 & 13 & $\ldots$ & $\ldots$ \\
\hline 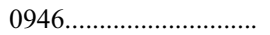 & 2.7 & 20 & 4.4 & 47 & $\ldots$ & $\ldots$ & $\ldots$ & $\ldots$ \\
\hline $1015 \ldots \ldots$ & 26 & 35 & 17 & 30 & 5.3 & 7.0 & 11 & 14 \\
\hline $1023 \ldots \ldots \ldots \ldots \ldots \ldots \ldots \ldots$ & 7.7 & 16 & 9.4 & 42 & $\ldots$ & $\ldots$ & $\ldots$ & $\ldots$ \\
\hline 1025 & 1.8 & 66 & 4.4 & 338 & $\cdots$ & $\cdots$ & $\cdots$ & $\cdots$ \\
\hline $1031 \ldots$ & 1.0 & 9.9 & 1.7 & 29 & $\cdots$ & $\cdots$ & $\cdots$ & $\cdots$ \\
\hline 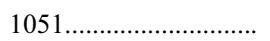 & 2.4 & 17 & 2.4 & 33 & 0.5 & 3.2 & 1.4 & 9.3 \\
\hline 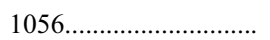 & 28 & 71 & 29 & 101 & 5.4 & 12 & 1.8 & 4.4 \\
\hline $1307 \ldots \ldots \ldots$ & 8.9 & 7.4 & 3.9 & 7.6 & 2.4 & 1.7 & 10 & 7.7 \\
\hline $1339 . \ldots \ldots$ & $\ldots$ & $\ldots$ & 8.6 & 76 & $\ldots$ & $\ldots$ & $\ldots$ & $\ldots$ \\
\hline $1429 \ldots \ldots$ & 9.3 & 24 & 5.6 & 31 & 2.7 & 6.4 & 6.9 & 19 \\
\hline 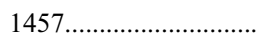 & 1.3 & 33 & 2.6 & 121 & $\ldots$ & $\ldots$ & $\ldots$ & $\ldots$ \\
\hline 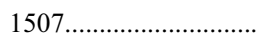 & $\ldots$ & $\ldots$ & 6.4 & 78 & $\ldots$ & $\ldots$ & $\ldots$ & $\ldots$ \\
\hline 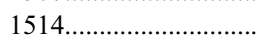 & 0.4 & 9.9 & 1.5 & 68 & $\ldots$ & $\ldots$ & $\ldots$ & $\ldots$ \\
\hline 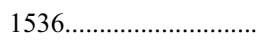 & 5.0 & 56 & 5.2 & 81 & 1.3 & 13 & $\ldots$ & $\ldots$ \\
\hline 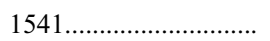 & 0.6 & 13 & 0.2 & 3.1 & $\ldots$ & $\ldots$ & $\ldots$ & $\ldots$ \\
\hline 1604 & 1.3 & 5.4 & 1.6 & 14 & $\cdots$ & 0.9 & 3.4 & $\cdots$ \\
\hline 1625 & 14 & 53 & 15 & 100 & 1.8 & 5.7 & 0.2 & 0.8 \\
\hline 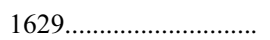 & 5.4 & 96 & 4.2 & 102 & 1.4 & 20 & 2.9 & 42 \\
\hline 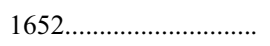 & 0.7 & 36 & 0.7 & 54 & $\ldots$ & $\ldots$ & $\ldots$ & $\ldots$ \\
\hline $1656 \ldots \ldots$ & 9.2 & 64 & 11 & 132 & 2.0 & 13 & $\ldots$ & $\ldots$ \\
\hline $2143 \ldots \ldots$ & 2.8 & 0.9 & 4.2 & 2.5 & $\ldots$ & $\ldots$ & $\ldots$ & $\ldots$ \\
\hline $2333 \ldots \ldots$ & 4.9 & 39 & 5.4 & 73 & 1,2 & 9.9 & 0.3 & 2.9 \\
\hline
\end{tabular}

${ }^{\text {a }}$ Fluxes are in units of $10^{-15} \mathrm{ergs}^{-2} \mathrm{~s}^{-1}$, and equivalent widths are in units of angstroms. 


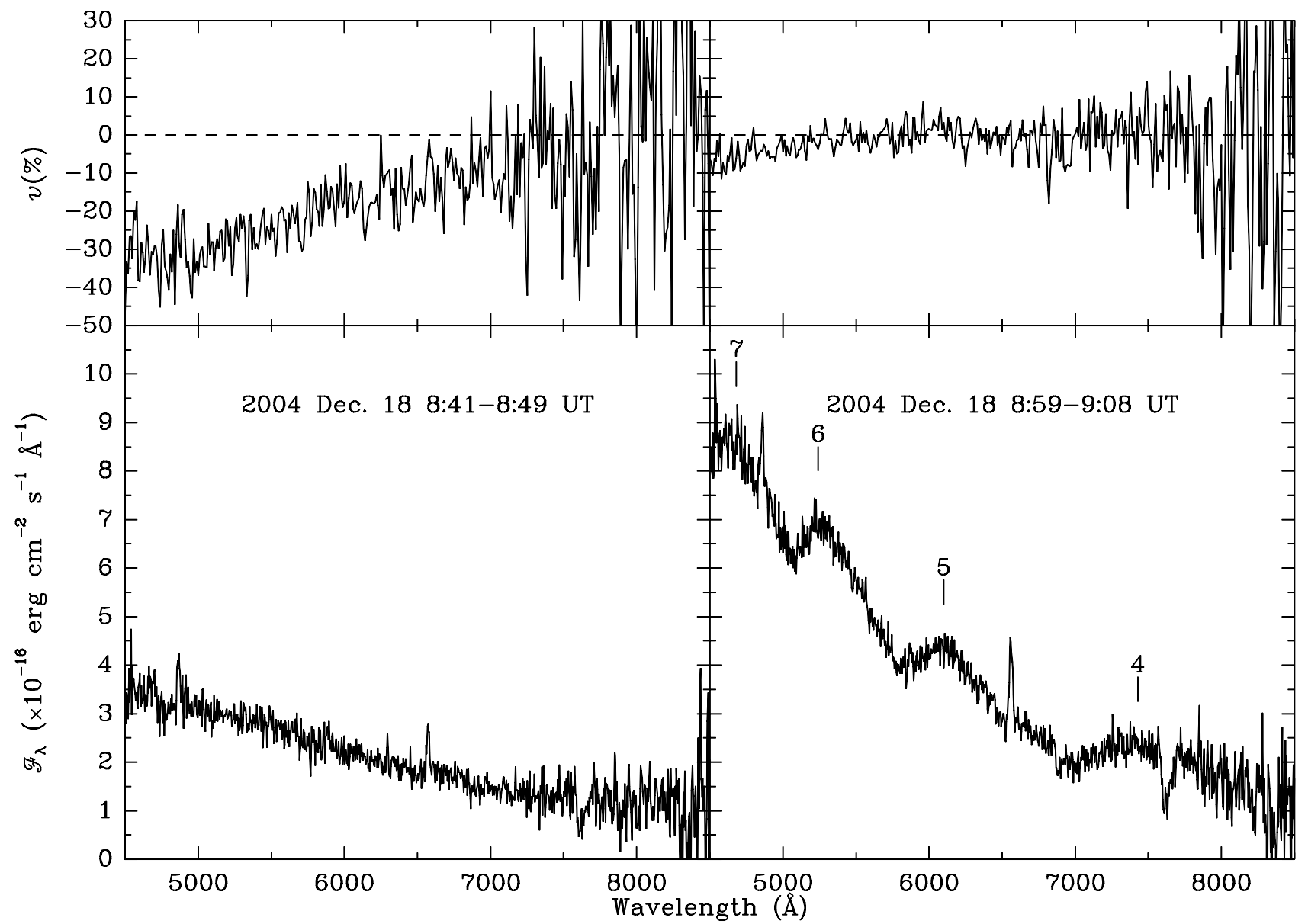

FIG. 2.-Circular polarization (top) and spectral flux (bottom) for the first (left) and third (right) observations of the polar SDSS J0854 (EUVE J0854+390). Numbers in the lower right spectrum are harmonics for a magnetic field of $44 \mathrm{MG}$.

TABLE 4

Radial Velocity Solutions

\begin{tabular}{|c|c|c|c|c|c|c|}
\hline SDSS J & Line & $\begin{array}{c}P \\
(\mathrm{hr})^{\mathrm{a}}\end{array}$ & $\gamma$ & $\begin{array}{c}K \\
\left(\mathrm{~km} \mathrm{~s}^{-1}\right)\end{array}$ & $T_{0}(\mathrm{JD} 2,450,000+)$ & $\sigma$ \\
\hline $0803 \ldots \ldots \ldots$ & $\mathrm{H} \alpha$ & 1.7 & $31 \pm 1$ & $67 \pm 5$ & 3002.725 & 8 \\
\hline $0803 \ldots \ldots$ & $\mathrm{H} \beta$ & 1.7 & $-35 \pm 1$ & $72 \pm 9$ & 3002.730 & 15 \\
\hline $0813 \ldots \ldots \ldots \ldots \ldots \ldots \ldots \ldots \ldots \ldots \ldots \ldots \ldots$ & $\mathrm{H} \alpha$ & 2.7 & $-44 \pm 1$ & $98 \pm 9$ & 2994.803 & 22 \\
\hline 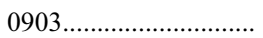 & $\mathrm{H} \alpha$ & 1.3 & $-13 \pm 3$ & $79 \pm 7$ & 3003.734 & 10 \\
\hline 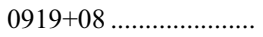 & $\mathrm{H} \alpha$ & 1.4 & $317 \pm 1$ & $67 \pm 5$ & 3112.660 & 11 \\
\hline $0919+08 .$. & $\mathrm{H} \beta$ & 1.4 & $166 \pm 2$ & $97 \pm 6$ & 3112.664 & 11 \\
\hline 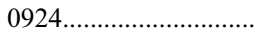 & $\mathrm{H} \alpha$ & 2.1 & $52 \pm 3$ & $345 \pm 21$ & 2994.919 & 33 \\
\hline 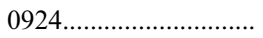 & $\mathrm{H} \beta$ & 2.0 & $128 \pm 3$ & $440 \pm 33$ & 2994.914 & 66 \\
\hline $0924 \ldots \ldots$. & $\mathrm{H} \gamma$ & 2.0 & $19 \pm 3$ & $450 \pm 34$ & 2994.915 & 67 \\
\hline 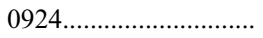 & He II & 2.1 & $91 \pm 8$ & $531 \pm 38$ & 2994.913 & 67 \\
\hline 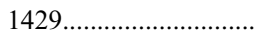 & $\mathrm{H} \alpha$ & 1.48 & $184 \pm 3$ & $84 \pm 13$ & 3055.906 & 20 \\
\hline 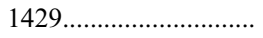 & $\mathrm{H} \beta$ & 1.35 & $127 \pm 2$ & $51 \pm 13$ & 3055.908 & 19 \\
\hline 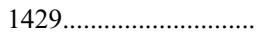 & $\mathrm{He}$ II & 1.45 & $71 \pm 2$ & $65 \pm 8$ & 3055.917 & 14 \\
\hline 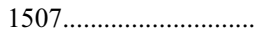 & $\mathrm{H} \alpha$ & $1.12 \mathrm{f}$ & $-30 \pm 2$ & $69 \pm 15$ & 2903.683 & 17 \\
\hline 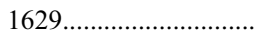 & $\mathrm{H} \alpha$ & $2.03 \mathrm{f}$ & $-26 \pm 1$ & $176 \pm 11$ & 2909.629 & 19 \\
\hline 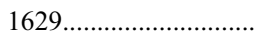 & $\mathrm{H} \beta$ & $2.03 \mathrm{f}$ & $200 \pm 2$ & $184 \pm 14$ & 2909.630 & 23 \\
\hline 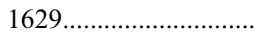 & $\mathrm{He}$ II & $2.03 \mathrm{f}$ & $0 \pm 5$ & $283 \pm 38$ & 2909.724 & 61 \\
\hline $2333 \ldots \ldots \ldots \ldots \ldots \ldots \ldots \ldots \ldots \ldots \ldots \ldots$ & $\mathrm{H} \alpha$ & 1.4 & $3 \pm 1$ & $96 \pm 14$ & 3261.695 & 38 \\
\hline 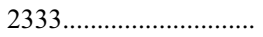 & $\mathrm{H} \beta$ & 1.35 & $-53 \pm 1$ & $99 \pm 16$ & 3261.697 & 46 \\
\hline
\end{tabular}

a An "f" following the period indicates the period was fixed at that value. 


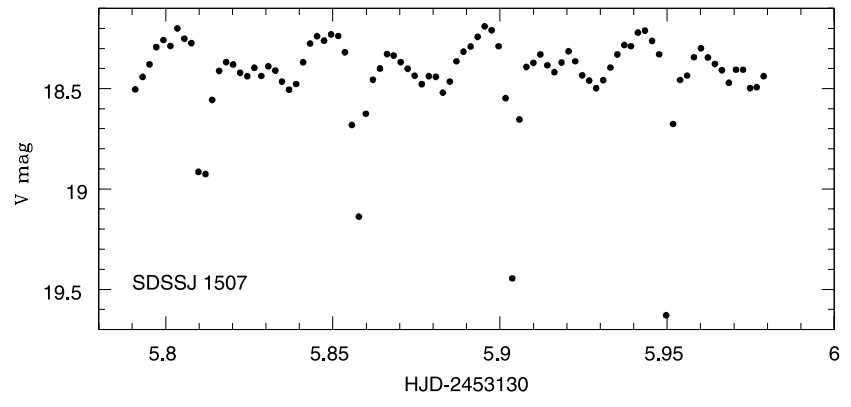

FIG. 3.- Light curve of the system SDSS J1507, showing four eclipses. Error bars are $0.02 \mathrm{mag}$, except during eclipses, when they become up to $0.05 \mathrm{mag}$.

that is typical of its low accretion rate during quiescence. Leo 7 shows the TiO bands from the secondary identified as an M3 V star by Thorstensen \& Taylor (2001) but no sign of the underlying white dwarf, indicating moderate rates of accretion similar to U Gem (which has a comparable orbital period). DV UMa also shows the M4.5 V secondary identified by Mukai et al. (1990), as well as the deep central absorption in the emission lines that is typical for a high-inclination eclipsing system.

Less information is known about EN Cet and UMa 8. The SDSS photometry and spectra improve on the limits given in the Downes catalog, but no periods are determined at this time.

\subsection{High-Inclination Systems}

On the basis of the very prominent central absorption in the Balmer emission lines (giving a double-peaked appearance) together with increasing absorption up the Balmer series, we identified five systems from Figure 1 as good candidates for having a high inclination. These include SDSS J0903, 0946, 1031,1457 , and 1507 . SDSS J0946 is the known eclipsing dwarf nova DV UMa described above. Our results on SDSS J0903 and 1507 are detailed below.

SDSS J0903.- The six APO spectra over $2.5 \mathrm{hr}$ have relatively long exposures of 15-20 minutes, which hinders the identification of short periods. The best solution was obtained for $\mathrm{H} \alpha$ using the double-Gaussian method (Table 4), which indicates an orbital period near $1.3 \mathrm{hr}$, but better time resolution and a longer data string are needed to determine this period accurately.

SDSS J1507.-Follow-up photometric observations identify an ultrashort orbital period of 67 minutes from observation of four consecutive eclipses (Fig. 3). The APO spectra cover this period twice, but the integration times are long (20-25 minutes) so that the velocity curve is not well resolved, resulting in a low $K$ amplitude for a high-inclination system (Table 4). The light curve shows a prominent hot spot preceding deep (>1.3 mag) eclipses. A larger telescope will be needed to resolve the eclipse structure and look for the secondary star during eclipse.

\subsection{Nova-like Systems with Strong He II}

The presence of strong He II emission lines usually indicates either a magnetic white dwarf or a nova-like system with a high level of mass transfer (an SW Sex star or an old nova). The presence of polarization confirms that a $\mathrm{CV}$ contains a magnetic white dwarf, but time-resolved photometry and spectroscopy can also provide strong clues. If an object has a large circular polarization and only shows a modulation of the lines and continuum at the orbital period, it is classified as an AM Her system, or polar (see review of polars in Wickramasinghe \& Ferrario 2000), and its spin is synchronized to its orbit. If it has little or no polarization and is observed to have a periodicity at a spin timescale of the white dwarf (minutes), it is considered to be an intermediate polar (IP), while if it has an orbital period between 3 and $4 \mathrm{hr}$ and single-peaked emission lines with central absorption appearing in the Balmer and $\mathrm{He}_{\mathrm{I}}$ lines, it is likely an SW Sex star (see Warner 1995 for a review of all types).

The systems SDSS J0018, 0813, 0837, 0854, 0859, 0924, 1015, 1051, 1307, 1429, 1541, 1604, and 1629 all show He II. SDSS $0859,1015,1051$, and 1307 are the previously known polars RX J0859, GG Leo, EK UMa, and EV UMa, and SDSS J0854 is EUVE J0854+390, which we confirmed as a polar, as described in $\S 3.1$. Spectropolarimetry was also accomplished for five of the new objects (SDSS J0018, 0837, 0924, 1541, and 1604). SDSS J0837 and 1541 are confirmed as polars from the presence of high circular polarization. No polarization was seen to a level of 0.2\% in SDSS J0018, 0.4\% in SDSS J0924, and to 0.1\% in SDSS J1604, so a polar nature is likely ruled out for these systems, unless the unobserved portions of the orbits are polarized. The time-resolved spectra of SDSS J0924 and 1629 show the signature narrow emission-line peaks and asymmetric broad bases that are associated with polars. SDSS J1429 shows some of the traits of an SW Sex star. The characteristics of the objects from available photometric and spectroscopic data are detailed below.

SDSS J0018.-Figure 1 shows that all the emission lines are very strong and narrow in this object. The lack of polarization rules out a polar nature, but the narrow lines do not resemble an SW Sex star either. The $2 \mathrm{hr}$ of APO spectra have good time resolution (10 minutes) and good $\mathrm{S} / \mathrm{N}$, yet all the velocities are constant to within $11 \mathrm{~km} \mathrm{~s}^{-1}$. The narrow lines and low velocities are indicative of a low-inclination disk system.

SDSS J0813.-The $3 \mathrm{hr}$ of APO spectra indicate an orbital period that is in the gap, near $2.7 \mathrm{hr}$ from the $\mathrm{H} \alpha$ solution (Table 4 and Fig. 4). The $\mathrm{H} \beta, \mathrm{H} \gamma$, and $\mathrm{He}$ II lines were noisier, so the periods were fixed at the value for $\mathrm{H} \alpha$ to try to determine solutions, but the resulting errors were still large. Further data will be needed to determine an accurate period for this system. It is interesting that this object is very faint on the Palomar Observatory Sky Survey (POSS) II $R$ plate but very bright on the $B$ plate and in the SDSS image and spectra. Since there are several years of difference between the POSS $B$ and $R$ plates, the object was likely in a low accretion state at the time of the $R$ plate.

SDSS J0837.- The SDSS spectrum (Fig. 1) of this object shows a broad blue hump and TiO bands from a secondary star. Polarimetry confirms the hump as a cyclotron feature (G. D. Schmidt et al. 2005, in preparation) and delegates this object as a member of the growing group of extremely low accretion rate

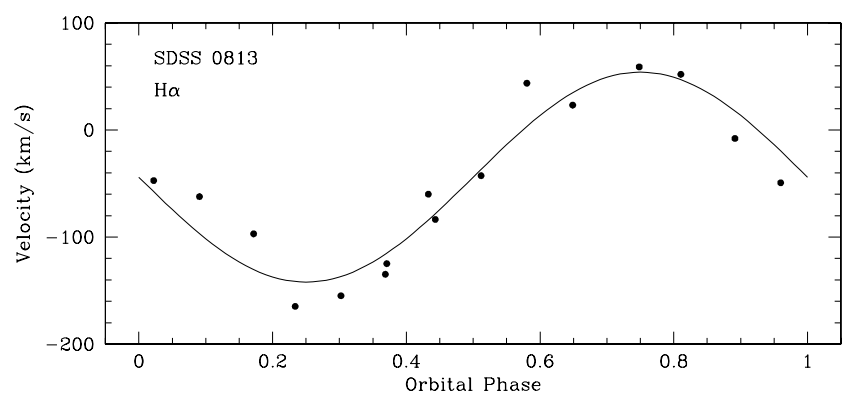

FIG. 4. - $\mathrm{H} \alpha$ velocity curve of SDSS J0813 with the best-fit sinusoid (Table 4). 


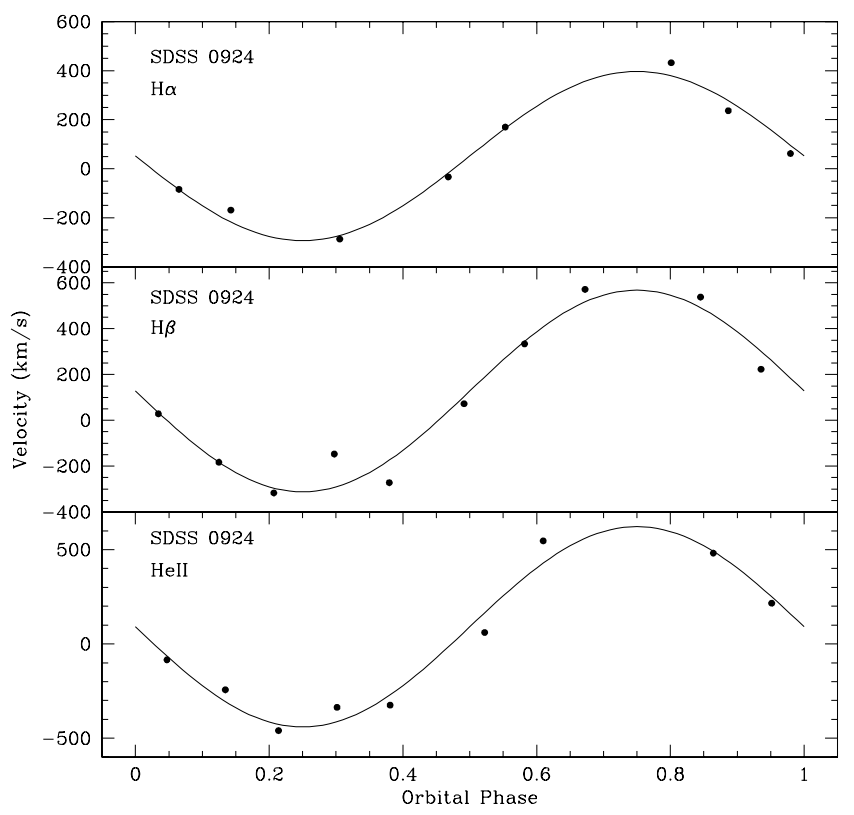

FIG. 5.-Velocity curves of SDSS J0924 with the best-fit sinusoids (Table 4).

systems such as SDSS J1553 and SDSS J1324 (Szkody et al. 2003a). No period is available.

SDSS J0924.- As in SDSS J0018, the SDSS spectrum (Fig. 1) reveals very narrow emission lines, and there are no polarization nor cyclotron humps evident during the MMT observation. The SDSS and APO spectra show little blue continuum, and the secondary is evident from $\mathrm{TiO}$ bands, indicating a very low accretion rate. It is puzzling that there is no evidence of a magnetic white dwarf even though the 11 time-resolved APO spectra reveal line structure that is typical of polars, i.e., narrow peaks and changing asymmetric broad bases throughout the $2 \mathrm{hr}$ of observation and the $K$ amplitude of the radial velocity curve is very large, as is typical for polars (Table 4 and Fig. 5). The photometry (Fig. 6) shows half-magnitude variations on a timescale near 65 minutes, roughly half of the orbital period of $2.1 \mathrm{hr}$ evident from the spectra. Phases on a $2.1 \mathrm{hr}$ period (with arbitrary zero) are shown at the top of Figure 6 . The variability is easiest to explain as a combination of obscuration from a mass transfer stream (near time 9.69 in Fig. 6) and a self-eclipse of an accreting pole (time 9.72-9.74). However, since the APO spectra only cover $2 \mathrm{hr}$ and the MMT polarimetry only 36 minutes, longer time intervals are needed for accurate period determination and to ensure that polarization is not present at any phase of the orbit.

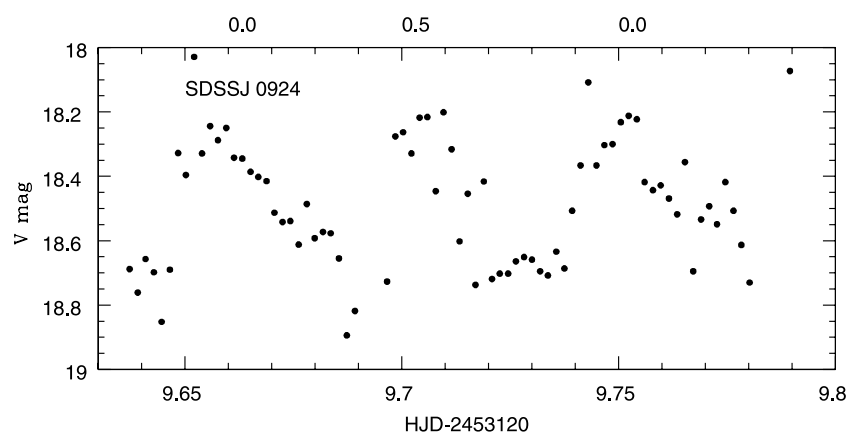

FIG. 6.- Light curve of SDSS J0924. Error bars on each point are 0.06 mag. Numbers at the top correspond to phasing on the $2.1 \mathrm{hr}$ period with an arbitrary zero point.

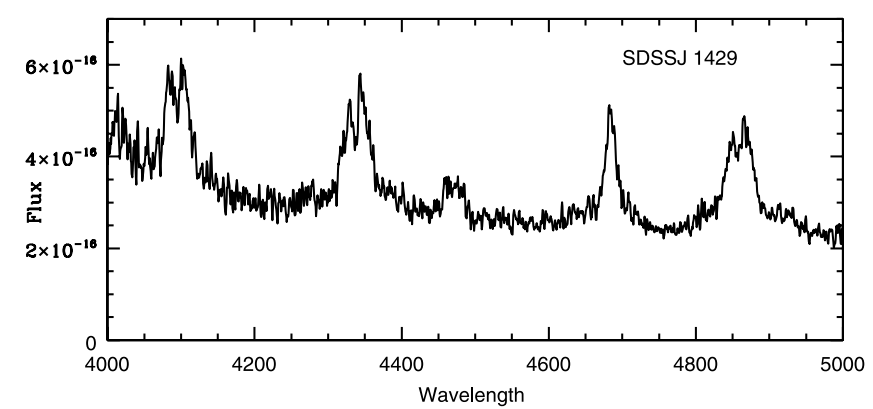

FIG. 7.-APO spectrum of SDSS J1429 showing the increased He II strength and absorption in the Balmer lines present during one of the seven spectra. The spectrum was of 15 minutes duration, with a midexposure time of 10:40 UT.

SDSS J1429.- - The SDSS spectrum (Fig. 1) shows strong single-peaked lines indicative of an SW Sex star. The seven time-resolved follow-up APO spectra are similar to the SDSS one, except for one spectrum (Fig. 7) with $\mathrm{He}$ II as strong as $\mathrm{H} \beta$ and with central absorption in the Balmer lines. This absorption, which is apparent only at some phases, is a typical signature of an SW Sex system. However, the radial velocities of $\mathrm{H} \alpha, \mathrm{H} \beta$, and $\mathrm{He}$ II all indicate an orbital period near $1.4 \mathrm{hr}$ (Table 4 and Fig. 8), whereas the SW Sex stars almost all have periods between 3-4 hr. However, since the 15-20 minutes integrations do not allow good phase resolution and the data only cover $2.5 \mathrm{hr}$, further work is needed to sort out the nature of this system.

SDSS J1541.-The SDSS spectrum of this object in Figure 1 shows a very low flux, with just a hint of the Balmer emission lines, He II, and a red continuum. However, followup spectropolarimetry found the system in a high-accretion state, with strong Balmer, $\mathrm{He}$ I, and $\mathrm{He}$ II emission lines, and circular polarization reaching $11 \%$ (Fig. 9): all features that are typical of a polar. Overlapping cyclotron harmonics are obvious in the spectral flux distribution and clearly shift to the red during the 17 minutes that separate the two observations. Fitting the peaks to the expected locations of cyclotron harmonics in a hot plasma yields the harmonic assignments indicated in the right panel of Figure 9, a magnetic field strength

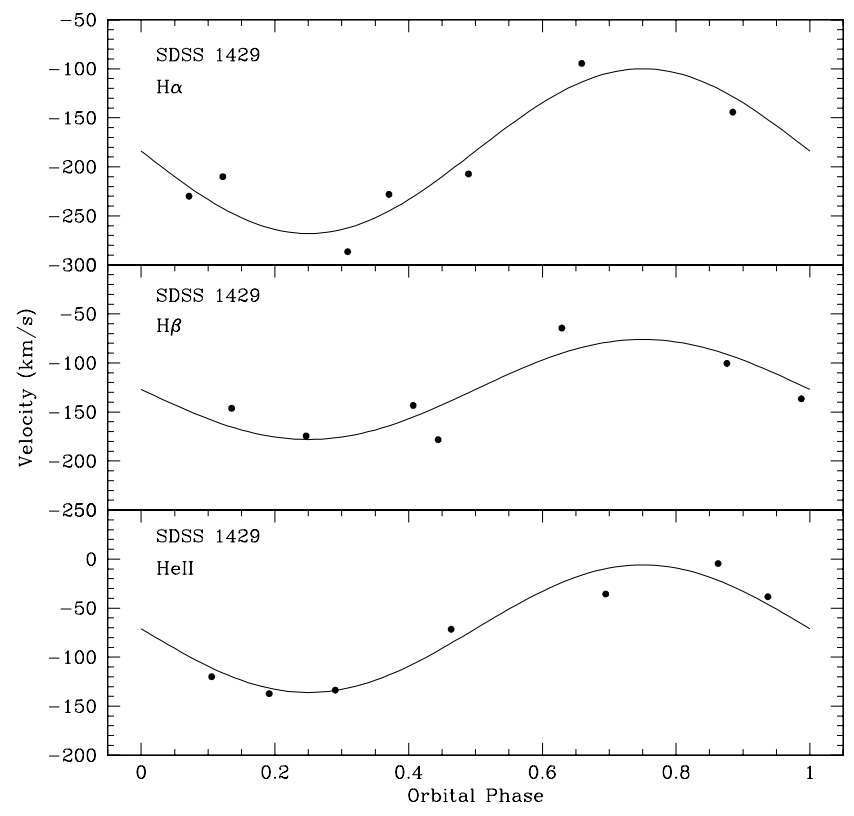

FIG. 8.-Velocity curves of SDSS J1429 with the best-fit sinusoids (Table 4). 


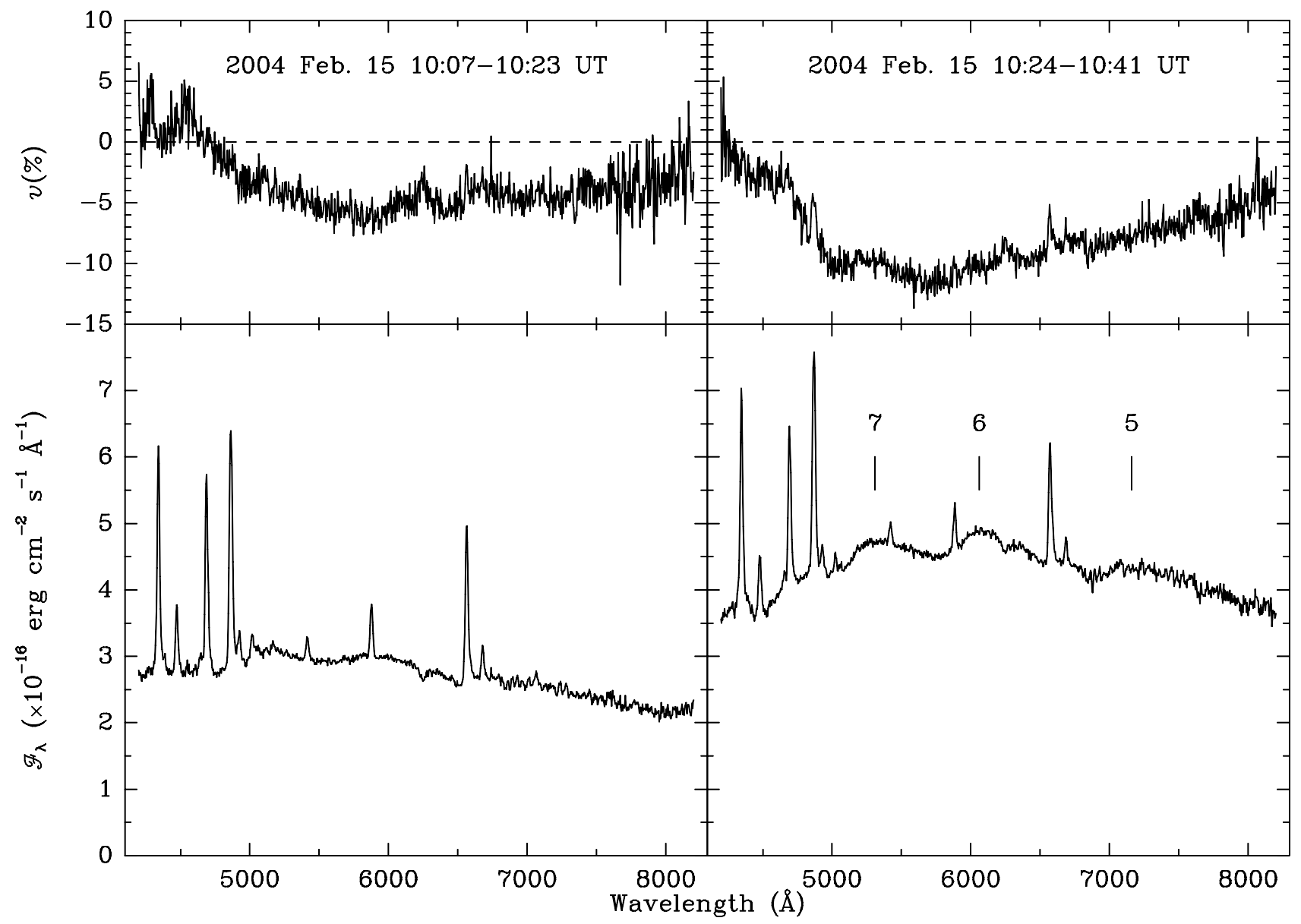

FIG. 9.-Circular polarization (top) and spectral flux (bottom) for the two observations of the polar SDSS J1541. Numbers in the bottom right spectrum indicate expected cyclotron harmonics for a field of $33 \mathrm{MG}$.

in the emission region $B=33 \mathrm{MG}$, and the temperature factor $T \sin ^{2} \theta$ changing from 4.6 to $7.1 \mathrm{keV}$ during the sequence. This result, the increased visibility of the harmonics, and the increased brightness between the two observations, imply a region that is being viewed with increasing inclination $\theta$ to the magnetic field. The light curve shown in Figure 10, also obtained during a high state, reveals a 1.3 mag modulation with a period of $1.4 \mathrm{hr}$, indicative of a geometry in which a single accretion pole is viewed for more than half the orbit and then selfeclipsed by the white dwarf for the rest. With such a short period, SDSS J1541 is clearly a target for more extensive observations.

SDSS J1629.- - This object has spectra very similar to SDSSJ 0924 in terms of showing narrow emission-line peaks and broad

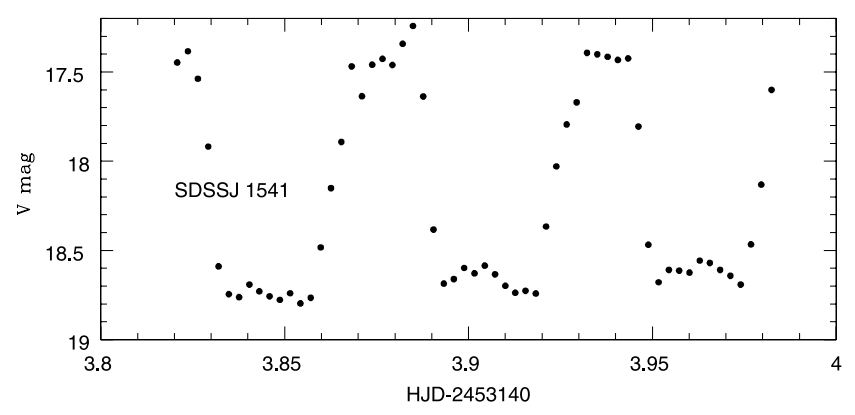

FIG. 10. - Light curve of SDSS J1541. Error bars on each point are $\leq 0.02$ mag. asymmetric wings during the follow-up APO spectra. The overall $\mathrm{He}$ II strength is much larger in SDSSJ 1629 than in SDSS J0924. As in SDSS J0924, the light curve of SDSS J1629 (Fig. 11) shows a large (0.6 mag) modulation, in this case more sharply defined and with a period of 122 minutes. The shape of the modulation is triangular, which would imply that the accretion column is always in view if this is indeed a polar. The APO spectra independently determine a period of 120 minutes from the velocities, but since the photometric period was better determined, the period was fixed at 122 minutes to obtain the best velocity solutions shown in Table 4 and Figure 12. As in SDSS J0924, the large $K$ amplitudes imply a polar nature for this object.

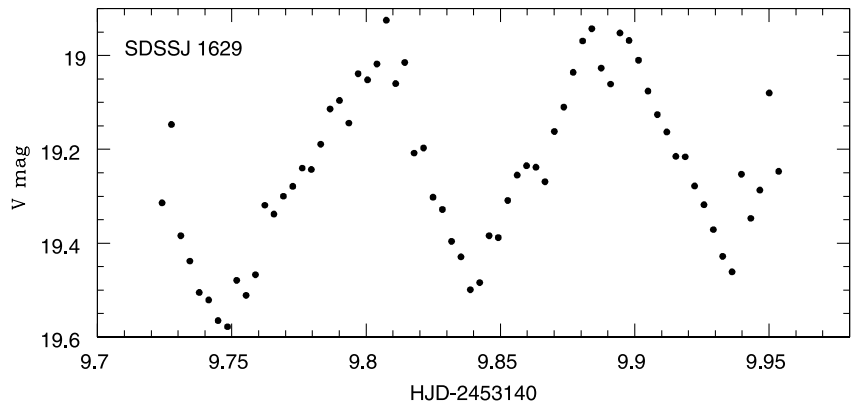

Fig. 11. - Light curve of SDSS J1629. Error bars are $0.02 \mathrm{mag}$. 


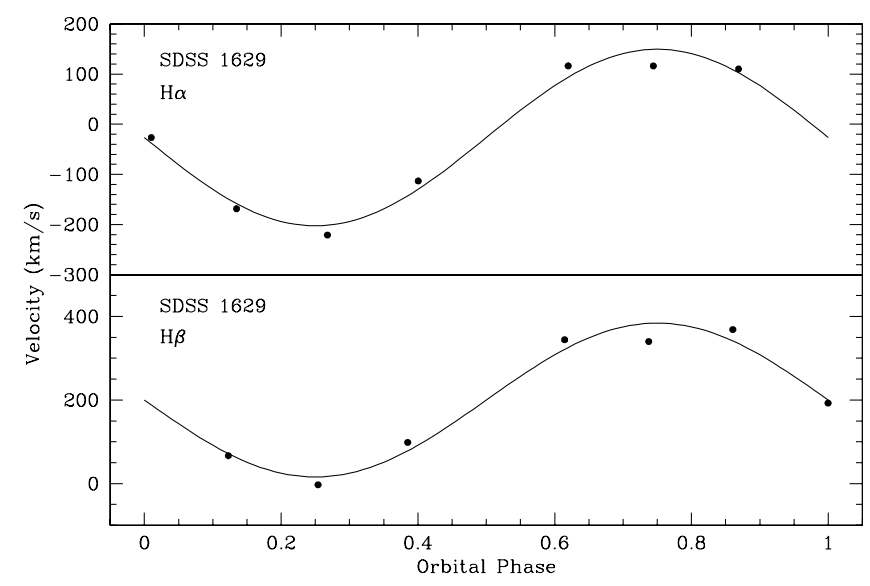

FIG. 12.-Velocity curves of SDSS J1629 with the best-fit sinusoids (Table 4).

\subsection{Systems Showing the Underlying Stars}

If the mass transfer rate is sufficiently low, the disks are tenuous and contribute little light compared to the primary and secondary stars. Thus, in these systems the white dwarf is evident through broad hydrogen absorption lines and the late $\mathrm{M}$ dwarf is evident from $\mathrm{TiO}$ bands and/or an upturn in the flux longward of $7000 \AA$. Figure 1 shows that there are 14 systems that show the underlying stars: SDSS J0843, 0856, 0919+08, $0922+42,1339,1457,1507$, and 1514 show the white dwarf, while SDSS J0837, 0859, 0906, 0911, 0919+50, 0924, and 0938 show features of an M star. SDSS J0859 is the previously known polar from ROSAT, SDSS J0837 is our newly confirmed polar, and SDSS J0924 is our candidate polar (all previously discussed in $\S 3.3$ ). SDSS J0906 is our likely dwarf nova ( $\S 3.5)$, SDSS J0938 is Leo 7 (§ 3.1), and SDSS J1507 is our new eclipsing system ( $\S 3.2)$.

The previous follow-up studies conducted by Woudt $\&$ Warner (2004) and Warner \& Woudt (2004) suggest that many of the systems showing the broad underlying absorption in the Balmer lines may contain pulsating white dwarfs.

SDSS J0919+08.- This system shows the prominent broad absorption lines from a white dwarf surrounding the $\mathrm{H} \beta$ and higher order Balmer emission lines. The double-Gaussian method with separations of $1400 \mathrm{~km} \mathrm{~s}^{-1}(\mathrm{H} \alpha)$ and $1500 \mathrm{~km}$ $\mathrm{s}^{-1}(\mathrm{H} \beta)$ gave consistent radial velocity solutions (Table 4 and Fig. 13), revealing a short orbital period of $1.4 \mathrm{hr}$. The spectrum

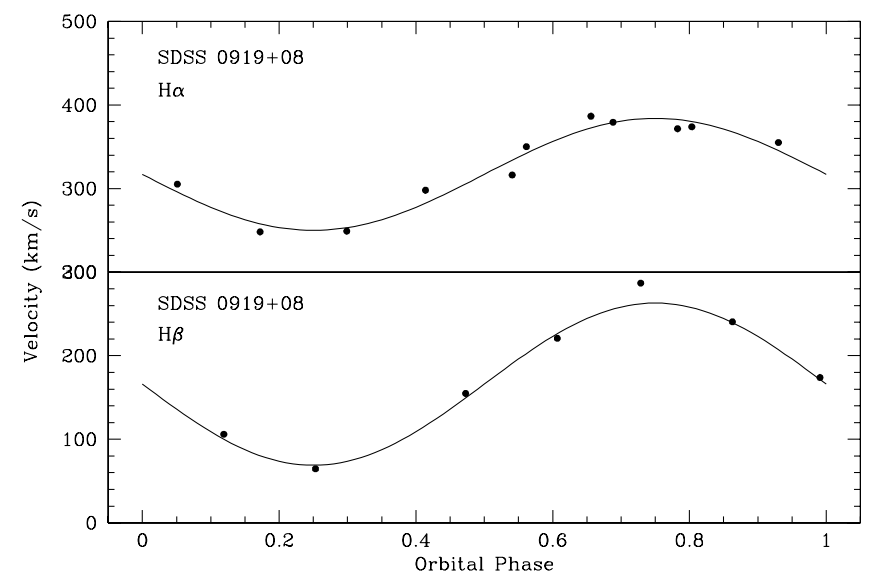

FIG. 13.- Velocity curves of SDSS J0919+08 with the best-fit sinusoids (Table 4).

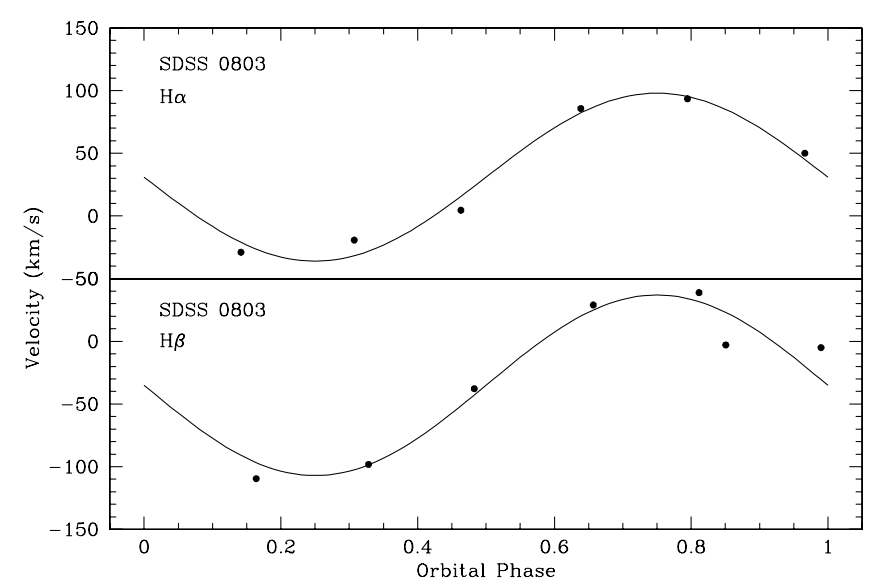

Fig. 14.-Velocity curves of SDSS J0803 with the best-fit sinusoids (Table 4).

and period are very similar to GW Lib, the first identified ZZ Ceti star in a CV (van Zyl et al. 2000).

SDSS J1339.- - Similar to SDSS J0919+08, prominent absorption from the white dwarf is apparent in the SDSS and APO spectra. However, in this case, no consistent solution could be obtained from the velocity curves, using either the doubleGaussian method on the line wings or the SPLOT centroids. In both methods, $\mathrm{H} \alpha$ gave periods near $1.7 \mathrm{hr}$, while $\mathrm{H} \beta$ and $\mathrm{H} \gamma$ gave solutions near $1.2 \mathrm{hr}$. A longer data set is needed to determine a good value for the period.

\subsection{Other Disk Systems}

While the SDSS spectrum and photometry of SDSS J0906 both indicate an object with a $g$-mag of 18.8 , the follow-up photometry at USNO caught the system at $V=15.5$, likely on the decline from an outburst, so this system is probably a dwarf nova. On the other hand, SDSS J2143 has a comparably large flux during the times of SDSS spectra and photometry, and the spectrum (Fig. 1) looks like a system in a high state of accretion, either near an outburst or like a nova-like system with a bright accretion disk. Since the fluxes are similar at different times of observation, the latter is more likely.

Follow-up observations were also accomplished for the systems SDSS J0801, 0803, and 2333, which appeared to be normal disk accreting systems from the SDSS spectra. While an estimate of the periods could be obtained for the latter two, the $2.5 \mathrm{hr}$ of 20 minutes spectra of SDSS J0801 were too noisy to

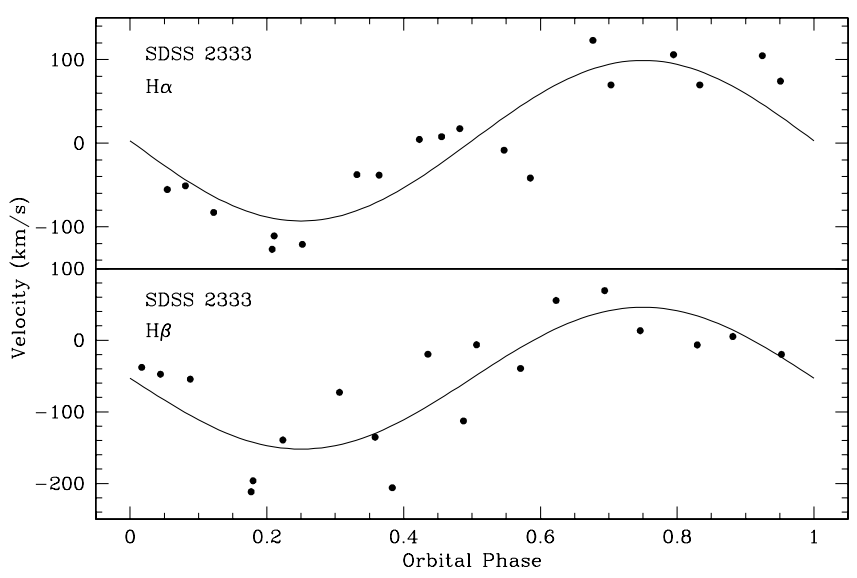

FIG. 15. - Velocity curves of SDSS J2333 with the best-fit sinusoids (Table 4). 


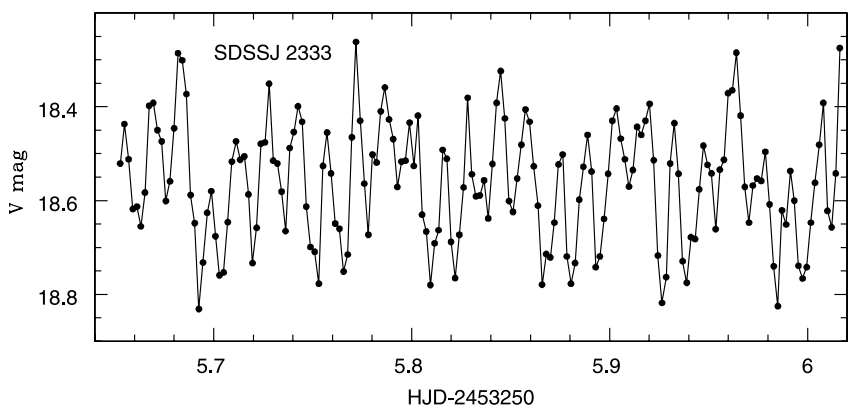

FIG. 16.- Light curve of SDSS J2333 showing the 21 minute sinusoidal periodicity. Error bars are $0.02 \mathrm{mag}$ on each point.

yield any consistent radial velocity solution for the $\mathrm{H} \alpha, \mathrm{H} \beta$, and $\mathrm{H} \gamma$ lines.

SDSS J0803.- Both the $\mathrm{H} \alpha$ and $\mathrm{H} \beta$ velocities show a sinusoidal curve with a period of $1.7 \mathrm{hr}$ (Table 4 and Fig. 14). This system appears to be a very typical disk-accreting CV.

$S D S S$ J2333. - The $3.5 \mathrm{hr}$ of APO spectra reveal velocity curves with a period of $1.4 \mathrm{hr}$ from both the $\mathrm{H} \alpha$ and $\mathrm{H} \beta$ emission lines (Table 4 and Fig. 15) and typical semiamplitudes near $100 \mathrm{~km}$ $\mathrm{s}^{-1}$. However, the spectra are noisy, consisting of 10 minute integrations in the attempt to resolve a short period. Surprisingly, the light curve (Fig. 16) reveals a periodicity at 21 minutes (determined from the PDM routine within IRAF) that is clearly visible as a modulation in the light curve with a peak-to-peak amplitude near $0.3 \mathrm{mag}$. The most plausible explanation is that we are seeing the spin period of the white dwarf, but if this is the case, then the ratio of spin to orbital period is much larger than the usual $10 \%$, and the amplitude of the spin modulation is unusually large. A longer spectroscopic study can pin down the errors on the orbital period and search for a spin modulation in the lines to confirm an IP nature.

\subsection{ROSAT Correlations}

Cross-correlating the CVs in Table 1 with the X-ray ROSAT All Sky Survey (RASS; Voges et al. 1999, 2000) shows nine with X-ray detections $(>2.5 \sigma)$ within the positional errors of the RASS. Table 5 gives the X-ray count rates for these sources. Included in Table 5 are the four previously known polars ( $\mathrm{RX}$ J0859+05, GG Leo, EK UMa, and EV UMa), the newly confirmed polar EUVE J0854+390, and the newly discovered polar SDSSJ 1541. The previously known bright dwarf novae CY UMa and V844 Her are also known RASS sources. It is not clear why SDSS J1656 has X-ray emission whereas systems of comparable optical brightness (implying similar distance and mass transfer rate) do not.

\section{CONCLUSIONS}

The $32 \mathrm{CVs}$ presented in this paper bring the total number of new CVs found in SDSS to 96. While the variety of systems seen covers the range of $\mathrm{CV}$ properties, it has become clear that SDSS is picking up a large number of extremely low mass transfer systems, including wind accretors (Paper I and III), as well as a large proportion of systems in which the underlying stars are viewed. The majority of the periods found in this group are less than $2 \mathrm{hr}$, yet there is not the pile up at or beyond the period minimum as predicted by the models of Howell et al. (2001). Thus, it appears that the correct prescriptions for angular momentum loss and selection effects are not yet known.

There are several interesting candidates deserving of further study in this group. SDSS J1507 has been found to show eclipses but the other systems that appear to be high inclination (SDSS J0903, 1031, and 1457) need to searched. Of the systems with prominent He II, SDSS J0837, 0854 (EUVE J0854+390), and 1541 are confirmed as polars, while two others (SDSS J0924 and 1629) show spectral features that indicate they are good candidates, and SDSS J1429 may be an SW Sex star. There are several systems containing possible pulsating white dwarfs (SDSS J0843, 0856, 0919+08, 0922+42, 1339, 1457, 1507, and 1514). Lastly, SDSS J2333 shows a 21 minute period in its light curve, while spectra indicate an orbital period near $1.4 \mathrm{hr}$. While the shorter period may be the spin timescale of the white dwarf, the ratio of spin to orbital timescales appears longer than usual.

Funding for the creation and distribution of the SDSS Archive has been provided by the Alfred P. Sloan Foundation, the Participating Institutions, NASA, the NSF, the US Department of Energy, the Japanese Monbukagakusho, and the Max Planck Society. The SDSS Web site is at http://www.sdss.org. The SDSS is managed by ARC for the Participating Institutions. The Participating Institutions are the University of Chicago, Fermilab, the Institute for Advanced Study, the Japan Participation Group, The Johns Hopkins University, the Korean Scientist Group, Los Alamos National Laboratory, the MaxPlanck-Institute for Astronomy, the Max-Planck-Institute for Astrophysics, New Mexico State University, University of Pittsburgh, Princeton University, the US Naval Observatory, and the University of Washington. P. S. and N. S. acknowledge support from NSF grant AST 02-05875. Studies of magnetic stars and stellar systems at Steward Observatory are supported by the NSF through grant AST 97-30792.

TABLE 5

ROSAT Detections

\begin{tabular}{|c|c|c|c|c|}
\hline SDSS $\mathrm{J}$ & $\begin{array}{c}\operatorname{ROSAT} \\
\left(\text { counts s }{ }^{-1}\right)^{\mathrm{a}}\end{array}$ & $\begin{array}{l}\text { Exposure } \\
\text { (s) }\end{array}$ & RXS & Type \\
\hline $0854 \ldots \ldots \ldots$ & $0.12 \pm 0.02$ & 420 & J085413.1+390543 = EUVE J0854 & Polar \\
\hline 0859 & $0.23 \pm 0.03$ & 366 & J085909.5+053703 & Polar \\
\hline 1015 & $1.15 \pm 0.10$ & 416 & $\mathrm{~J} 101534.8+090448=$ GG Leo & Polar \\
\hline $1051 \ldots \ldots \ldots \ldots$ & $1.11 \pm 0.04$ & 600 & $\mathrm{~J} 105135.3+540438=\mathrm{EK} \mathrm{UMa}$ & Polar \\
\hline 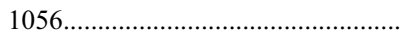 & $0.096 \pm 0.016$ & 523 & $\mathrm{~J} 105656.9+494111=\mathrm{CY} \mathrm{UMa}$ & $\mathrm{DN}$ \\
\hline $1307 \ldots \ldots \ldots$ & $1.86 \pm 0.06$ & 484 & $\mathrm{~J} 130753.6+535137=\mathrm{EV} \mathrm{UMa}$ & Polar \\
\hline 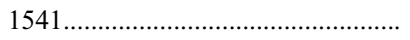 & $0.05 \pm 0.01$ & 370 & $\mathrm{~J} 154104.6+360252$ & Polar \\
\hline 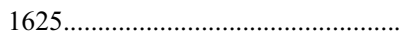 & $0.08 \pm 0.01$ & 857 & $\mathrm{~J} 162501.2+390924=\mathrm{V} 844 \mathrm{Her}$ & $\mathrm{DN}$ \\
\hline 1656 & $0.04 \pm 0.01$ & 629 & $\mathrm{~J} 165658.7+212141$ & $\ldots$ \\
\hline
\end{tabular}

\footnotetext{
${ }^{\text {a }}$ For a $2 \mathrm{keV}$ bremsstrahlung spectrum, 1 count s ${ }^{-1}$ corresponds to a $0.1-2.4 \mathrm{keV}$ flux of about $7 \times 10^{-12} \mathrm{ergs} \mathrm{cm}^{-2} \mathrm{~s}^{-1}$.
} 
Abazajian, K., et al. 2003, AJ, 126, 2081 (DR1) . 2004, AJ, 128, 502 (DR2) 2005, AJ, 129, 1755 (DR3)

Christian, D. J., Craig, N., Dupuis, J., Roberts, B. A., \& Malina, R. F. 2001, AJ, 122,378

Downes, R. A., Webbink, R. F., Shara, M. M., Ritter, A., Kolb, U., \& Duerbeck, H. W. 2001, PASP, 113, 764

Fukugita, M., Ichikawa, T., Gunn, J. E., Doi, M., Shimasaku, K., \& Schneider, D. P. 1996, AJ, 111, 1748

Gänsicke, B. T. 2005, in ASP Conf. Ser. 330, The Astrophysics of Cataclysmic Variables and Related Objects, ed. J.-M. Hameury \& J.-P. Lasota (San Francisco: ASP), in press

Gunn, J. E., et al. 1998, AJ, 116, 3040

Hogg, D. W., Finkbeiner, D. P., Schlegel, D. J., \& Gunn, J. E. 2001, AJ, 122, 2129

Howell, S. B., Nelson, L. A., \& Rappaport, S. 2001, ApJ, 550, 897

Ivezic, Z., et al. 2004, Astron. Nachr., 325, 583

Lupton, R. H., Gunn, J. E., Ivezic, Z., Knapp, G. R., Kent, S. M., \& Yasuda, N. 2001, in ASP Conf. Ser. 238, Astronomical Data Analysis Software and Systems X, ed. F. R. Harnden, Jr., et al. (San Francisco: ASP), 269

Lupton, R. H., Gunn, J. E., \& Szalay, A. 1999, AJ, 118, 1406

Mukai, K., et al. 1990, MNRAS, 245, 385

\section{REFERENCES}

Pier, J. R., Munn, J. A., Hindsley, R. B., Hennessy, G. S., Kent, S. M., Lupton, R. H., \& Ivezic, Z. 2003, AJ, 125, 1559

Shafter, A. W. 1983, ApJ, 267, 222

Smith, J. A., et al. 2002, AJ, 123, 485

Stoughton, C., et al. 2002, AJ, 123, 485

Szkody, P., et al. 2002, AJ, 123, 430 (Paper I)

. 2003a, ApJ, 583, 902

$$
\text { 2003b, AJ, 126, } 1499 \text { (Paper II) }
$$

- 2004, AJ, 128, 1882 (Paper III)

Thorstensen, J. R., \& Taylor, C. J. 2001, MNRAS, 326, 1235

van Zyl, L., Warner, B., O’Donoghue, D., Sullivan, D., Pritchard, J., \& Kemp, J. 2000, Baltic Astron., 9, 231

Voges, W., et al. 1999, A\&A, 349, 389 2000, IAU Circ., 7432

Warner, B. 1995, Cataclysmic Variable Stars (Cambridge: Cambridge Univ. Press)

Warner, B., \& Woudt, P. 2004, in ASP Conf. Ser. 310, Variable Stars in the Local Group, ed. D. Kurtz \& K. Pollard (San Francisco: ASP), 382

Wickramasinghe, D. T., \& Ferrario, L. 2000, PASP, 112, 873

Woudt, P., \& Warner, B. 2004, MNRAS, 348, 599

York, D. G., et al. 2000, AJ, 120, 1579 